\title{
Nonequilibrium GW approach to quantum transport in nano-scale contacts
}

\author{
Kristian S. Thygesen \\ Institut für Theoretische Physik, Freie Universität Berlin, Arnimallee 14, D-14195 Berlin, Germany \\ Angel Rubio \\ Departamento de Física de Materiales, Facultad de Ciencias Químicas, University of the Basque Country \\ UPV/EHU, Centro Mixto and European Theoretical Spectroscopy Facility (ETSF), \\ E-20018 San Sebastián, Basque Country, Spain
}

(Received 30 November 2006; accepted 12 January 2007; published online 2 March 2007)

\begin{abstract}
Correlation effects within the $G W$ approximation have been incorporated into the Keldysh nonequilibrium transport formalism. We show that $G W$ describes the Kondo effect and the zero-temperature transport properties of the Anderson model fairly well. Combining the $G W$ scheme with density functional theory and a Wannier function basis set, we illustrate the impact of correlations by computing the $I-V$ characteristics of a hydrogen molecule between two Pt chains. Our results indicate that self-consistency is fundamental for the calculated currents, but that it tends to wash out satellite structures in the spectral function. () 2007 American Institute of Physics.

[DOI: $10.1063 / 1.2565690]$
\end{abstract}

Electronic correlations are responsible for important transport phenomena such as Coulomb blockade and Kondo effects, ${ }^{1}$ yet its significance for transport in nanoscale structures is not well understood nor has it been systematically studied. At present, the most popular approach to ab initio simulations of transport in nanocontacts combines a nonequilibrium Green's function formalism with the single-particle Kohn-Sham (KS) scheme of density functional theory (DFT). This approach works well for some systems, ${ }^{2,3}$ but in other cases it fails to reproduce experimental data ${ }^{4}$ indicating the need for computational transport schemes beyond the DFT level. ${ }^{5-7}$

A reliable description of transport through a molecular junction requires first of all a reliable description of the electronic structure of the molecule itself, i.e., its electron addition and removal energies. It is well known that the $G W$ self-energy method yields quasiparticle properties of molecules ${ }^{8,9}$ and solids ${ }^{10,11}$ in good agreement with experiment improving drastically the DFT band structures. In view of this it seems tempting to extend the use of the $G W$ approximation to transport calculations. It is clear, however, that this should not be implemented by shifting the molecular energy levels to their $G W$ positions prior to coupling. The reason is, that when a confined interacting system is connected to external (non-interacting) leads, the electrons in the confined region become correlated with those in the leads. To capture these correlations, which are the origin of important many-body phenomena such as the Kondo effect, it is crucial that the self-energy be evaluated in the presence of coupling to the leads.

Traditionally, correlation effects in transport have been studied on the basis of the Anderson and Kondo models by a variety of numerical and analytical techniques. Many of these techniques are, however, quite specific to the considered models and lack the generality needed to be combined with first-principles methods.

In this paper we combine the $G W$ approximation with the nonequilibrium Keldysh formalism to obtain a practical scheme for correlated quantum transport. We study the Anderson model out of equilibrium, and calculate the $I-V$ characteristics of a molecular hydrogen contact using a Wannier function (WF) basis set. In both applications we emphasize the difference between self-consistent and nonselfconsistent evaluations of the $G W$ self-energy.

As a general model of a quantum conductor we consider a central region $(C)$ connected to left $(L)$ and right $(R)$ leads. The leads are kept at chemical potentials $\mu_{L}$ and $\mu_{R}$, respectively. We construct the matrix $h_{i j}$ $=\left\langle\phi_{i}\left|\hat{h}_{s}\right| \phi_{j}\right\rangle$, where $\hat{h}_{s}=-\frac{1}{2} \nabla^{2}+v_{h}+v_{x c}+v_{\text {ext }}$ is the KS Hamiltonian of the combined $L-C-R$ system in equilibrium, and $\left\{\phi_{i}\right\}$ is a corresponding set of maximally localized, partly occupied WFs. ${ }^{12}$ Assuming that correlation effects as well as charge redistributions induced by the bias voltage are significant only inside $C$, we describe the leads and the coupling to the central region by $h$. Using standard methods ${ }^{13}$ we evaluate the coupling self-energies, $\Sigma_{\alpha}(\omega)=h_{C \alpha} g_{\alpha \alpha}(\omega) h_{\alpha C}$, where $g_{\alpha \alpha}$ is the GF of the uncoupled lead $\alpha=L, R$. The interactions inside the central region are described by $\hat{V}_{\text {int }}$ $=\frac{1}{2} \sum_{i j k l \in C, \sigma \sigma^{\prime}} V_{i j, k l} c_{i \sigma^{\prime}}^{\dagger} c_{j \sigma^{\prime}}^{\dagger} c_{l \sigma^{\prime}} c_{k \sigma}$, with the Coulomb matrix elements $V_{i j, k l}=\iint \mathrm{d} \mathbf{r} \mathrm{d} \mathbf{r}^{\prime} \phi_{i}(\mathbf{r})^{*} \phi_{j}\left(\mathbf{r}^{\prime}\right)^{*} \phi_{k}(\mathbf{r}) \phi_{l}\left(\mathbf{r}^{\prime}\right) /\left|\mathbf{r}-\mathbf{r}^{\prime}\right|$. In practice we use an effective interaction involving only a subset of the Coulomb matrix elements (see later). We include matrix elements of the form $V_{i j, i j}$ and $V_{i j, j i}$ in the calculation of the correlation part of the $G W$ self-energy while also terms of the form $V_{i i, j j}$ and $V_{i i, i j}$ are included in the Hartree and exchange self-energies. ${ }^{14}$ By allowing the effective interaction to be spin-dependent we can avoid the self-interaction otherwise present in the correlation part of the $G W$ selfenergy.

We evaluate the retarded and lesser Green's functions of the central region from ${ }^{15,16}$

$$
G^{r}=\left[\omega+i \eta-h_{C C}+\left(v_{x c}\right)_{C C}-\Delta v_{h}-\Sigma_{\text {tot }}^{r}\right]^{-1},
$$




$$
G^{<}=G^{r} \Sigma_{\text {tot }}^{<} G^{a}+2 \eta i f_{C} G^{r} G^{a},
$$

where $\eta$ is a positive infinitesimal and $\Sigma_{\text {tot }}=\Sigma_{L}+\Sigma_{R}+\Sigma$ is the sum of the coupling self-energies and the exchangecorrelation part of the interaction self-energy. The term $\Delta v_{h}$ $\equiv \Sigma_{h}^{r}[G]-\sum_{h}^{r}\left[G_{\mathrm{DFT}}^{\mathrm{eq}}\right]$, is the correction to the equilibrium DFT Hartree potential introduced by the correlations and the finite bias. The DFT xc-potential is subtracted from $h_{C C}$ to avoid double counting. In the last term of Eq. (2), $f_{C}$ denotes the initial Fermi-Dirac distribution of the central region before coupling to the leads. Notice that this term can become significant at energies where $\operatorname{Im} \Sigma_{\text {tot }}^{r}$ is comparable to $\eta$. $^{16}$ While this is not expected to occur in the interacting case (due to lifetime broadening by $\Sigma$ ), it will happen in the noninteracting case whenever bound states are present in $C$. We note, that in Eqs. (1) and (2) we have specialized to the long time limit where we assume that the system reaches a steady state in which the Green's functions depend only on the time difference $t=t_{2}-t_{1}$, and thus, can be represented by a single time/frequency variable. ${ }^{17}$

The symmetrized current, $I=\left(I_{L}+I_{R}\right) / 2$, where $I_{L(R)}$ is the current in the left (right) lead, is given by ${ }^{18}$

$$
I=\frac{i}{4 \pi} \int \operatorname{Tr}\left[\left(\Gamma_{L}-\Gamma_{R}\right) G^{<}+\left(f_{L} \Gamma_{L}-f_{R} \Gamma_{R}\right)\left(G^{r}-G^{a}\right)\right] \mathrm{d} \omega,
$$

where $\Gamma_{L(R)}=i\left[\Sigma_{L(R)}^{r}-\Sigma_{L(R)}^{a}\right]$ is the coupling strength and the trace is taken over basis functions in the central region.

Within the $G W$ approximation $\Sigma$ is written as a product of the Green's function, $G$, and the screened interaction, $W$, calculated in the random-phase approximation (RPA). Out of equilibrium this holds true on the Keldysh contour, and the relevant equations in real time follow from the Langreth conversion rules. ${ }^{15}$ Absorbing the spin index into the orbital index we define the effective interaction $\hat{V}_{\text {eff }}=\frac{1}{2} \Sigma_{i j} \widetilde{V}_{i j} c_{i}^{\dagger} c_{j}^{\dagger} c_{j} c_{i}$, where $\widetilde{V}_{i j}=V_{i j, i j}-\delta_{\sigma \sigma^{\prime}} V_{i j, j i}$. $\hat{V}_{\text {eff }}$ resembles the real space interaction with the important difference that $\widetilde{V}_{i j}$ is spindependent and $V_{i i}=0$. Self-interaction is thus automatically excluded to all orders in $\hat{V}_{\text {eff }}$. The retarded and lesser $G W$ self-energies become (on the time axis),

$$
\begin{aligned}
& \Sigma_{i j}^{r}(t)=i G_{i j}^{r}(t) W_{i j}^{>}(t)+i G_{i j}^{<}(t) W_{i j}^{r}(t), \\
& \Sigma_{i j}^{<}(t)=i G_{i j}^{<}(t) W_{i j}^{<}(t) .
\end{aligned}
$$

The screened interaction is given by (in frequency space),

$$
\begin{aligned}
& W^{r}(\omega)=\tilde{V}\left[I-P^{r}(\omega) \tilde{V}\right]^{-1}, \\
& W^{<1>}(\omega)=W^{r}(\omega) P^{<1>}(\omega) W^{a}(\omega),
\end{aligned}
$$

where all quantities are matrices in the central region indices. Finally the irreducible polarization becomes

$$
\begin{aligned}
& P_{i j}^{r}(t)=-i G_{i j}^{r}(t) G_{j i}^{<}(-t)-i G_{i j}^{<}(t) G_{j i}^{a}(-t), \\
& P_{i j}^{<>>}(t)=-i G_{i j}^{</>}(t) G_{j i}^{>l<}(-t) .
\end{aligned}
$$

In principle the $G W$ method implies a self-consistent problem, i.e., the $G$ obtained from Eqs. (1) and (2) should equal the $G$ used to produce $\Sigma$. In this way all important conservation laws are fulfilled. ${ }^{19}$ However, due to the large

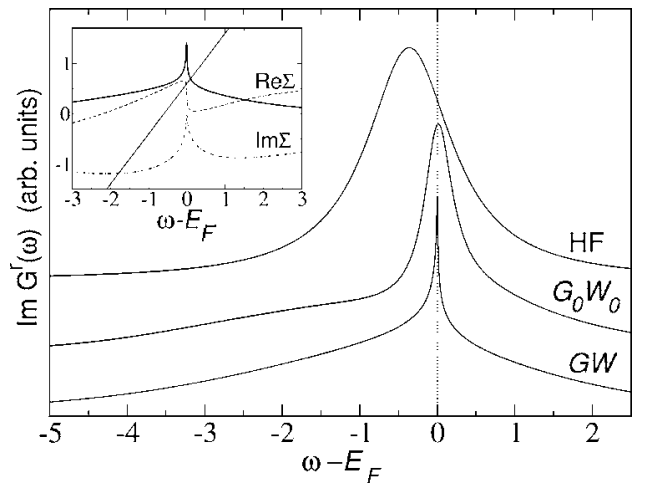

FIG. 1. Spectral function at the central site, $\varepsilon_{c}=-3$, of the Anderson model calculated in three different ways (see text). The curves have been vertically offset for clarity. Inset: real and imaginary parts of the $G W$ self-energy together with the line $\omega-\varepsilon_{\mathrm{HF}}$. The steep shape of $\operatorname{Re} \Sigma^{r}$ around $E_{F}$ pins the position of the quasi-particle peak.

computational demands $G W$ band structure calculations usually apply a, quite successful, non self-consistent $G_{0} W_{0}$ approach using on the $G_{0}$ obtained from a KS calculation. ${ }^{10} \mathrm{We}$ mention here that the nonequilibrium $G W$ approximation has previously been used in the study of semiconductors in highintensity laser fields. ${ }^{15,20}$

We represent all quantities on a uniform real time/ frequency grid using the fast Fourier transform to switch between the two representations to avoid time consuming convolutions. The calculation of the WFs, the matrix elements $h_{i j}$, and the coupling self-energies $\Sigma_{\alpha}$ is described in detail in Ref. 13.

Before turning to the Wannier- $G W$ calculations we apply the method to the Anderson impurity model. Although $G W$ is not expected to be accurate for strongly correlated systems, the simplicity of the Anderson model makes it ideal for illustrating the properties and limitations of the nonequilibrium $G W$ approximation. We thus consider a central site of energy $\varepsilon_{c}$ coupled to one-dimensional leads with on-site energy, $\varepsilon_{0}=0$, and nearest-neighbor hopping $t_{0}=10$. The hopping to the central site is $t_{c}=1.8$ giving $\Gamma \equiv \Gamma_{L}(0)=\Gamma_{R}(0)$ $\approx 0.65$. Double occupation of the central site costs a charging energy of $U=4$, and the spin-dependent interaction entering Eq. (5) is $\widetilde{V}_{\sigma \sigma^{\prime}}=U\left(1-\delta_{\sigma \sigma^{\prime}}\right)$. We assume half-filled bands, i.e., $E_{F}=\varepsilon_{0}$, and measure all energies relative to $E_{F}$.

We consider three different approximations. (i) Selfconsistent Hartree, which is equivalent to self-consistent Hartree-Fock (HF) since the exchange term vanishes. (ii) $G_{0} W_{0}$ with the self-consistent HF Green's funtion as $G_{0}$. (iii) Fully self-consistent $G W$. All calculations are nonmagnetic, i.e., $G_{\uparrow}=G_{\downarrow}=G$.

In Fig. 1 we show the equilibrium spectral function at the central site, $\operatorname{Im} G^{r}(\omega)$, for $\varepsilon_{c}=-3$. The HF solution shows a single peak at $\varepsilon_{\mathrm{HF}}=\varepsilon_{c}+U\left\langle\hat{n}_{\sigma}\right\rangle$ with a full width at half maximum given by $2 \Gamma$. This behavior is representative for any mean-field description, including the KS scheme. The inclusion of dynamic correlations leads to qualitative changes in the spectral peak which moves close to the chemical potential ${ }^{21}$ and narrows down from $2 \Gamma$ to 0.63 $(0.28)$ in the case of $G_{0} W_{0}(G W)$. This change is a signature of the Kondo effect: For $\Gamma-U<\varepsilon_{c}<-\Gamma$ (the so-called 


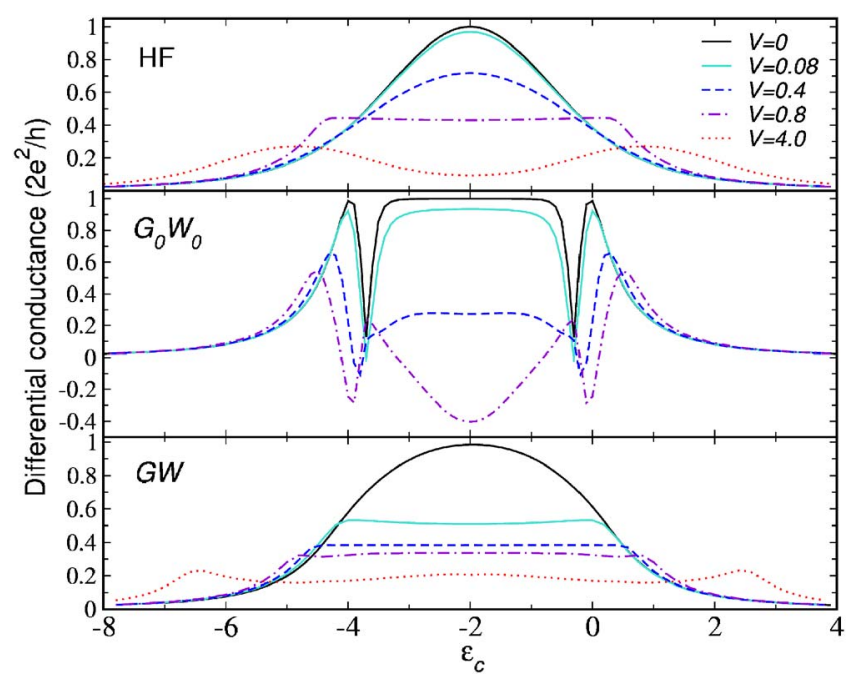

FIG. 2. (Color online) Differential conductance in the Anderson impurity model as a function of the central site energy, $\varepsilon_{c}$, for different applied biases. The negative differential conductance seen in the middle panel is an artifact of the nonconserving nature of the $G_{0} W_{0}$ approximation.

Kondo regime), the correlated groundstate is a singlet with a finite amplitude for the central site being empty. At $T=0$ this leads to the formation of a spectral peak at the chemical potential with a width given by the Kondo temperature, $T_{K}$ $=\frac{1}{2}(2 \Gamma U)^{1 / 2} \exp \left[\pi \varepsilon_{c}\left(\varepsilon_{c}+U\right) / 2 \Gamma U\right]$. For our choice of parameters $T_{K}=0.19$, which is in fair agreement with the $G W$ result, and about three times smaller than the $G_{0} W_{0}$ result.

From the inset of Fig. 1 it can be seen that the Kondo peak gets pinned to $E_{F}$ due to the steep shape of $\operatorname{Re} \Sigma^{r}$ in this region and that its reduced width, as compared to $2 \Gamma$, is a consequence of the steep drop in $\operatorname{Im} \Sigma^{r}$ away from the point $\operatorname{Im} \Sigma^{r}\left(\omega=E_{F}\right)=0$. The atomic levels which should be seen at $\varepsilon_{c}$ and $\varepsilon_{c}+U$ appear as shoulders in the $G_{0} W_{0}$ spectrum, but for larger values of $U / \Gamma$ they become more pronounced as satellites (sidebands) of the main quasiparticle peak although their positions are somewhat shifted towards $E_{F}$. In contrast the self-consistent $G W$ fails to capture the sidebands. These findings agree well with previous results obtained with the fluctuation-exchange approximation, ${ }^{22}$ and with $G W$ studies of the homogeneous electron gas. ${ }^{23}$ We mention that in selfconsistent second-order perturbation theory, the pinning of the main spectral peak to $E_{F}$ is less pronounced than in $G W$ and its width is significantly overestimated, showing as expected that the higher order RPA diagrams enhance the strong correlation features.

In Fig. 2 we show the zero-temperature differential conductance under a symmetric bias, $\mu_{L / R}= \pm V / 2$, as a function of $\varepsilon_{c}$. For $V=0$ there is only little difference between the HF and $G W$ results which both shows a broad conductance peak reaching the unitary limit at the symmetric point $\varepsilon_{c}=-U / 2$. The physical origin of the conductance trace is, however, very different in the two cases. While the HF result is produced by off-resonant transport through a broad spectral peak moving rigidly through the Fermi level, the $G W$ result is due to transport through a narrow Kondo peak which is always on resonance (for $\varepsilon_{c}$ in the Kondo regime). This difference is brought out clearly as $V$ is increased: For $V \ll \Gamma$ the bias has little effect on the HF conductance while the $G W$

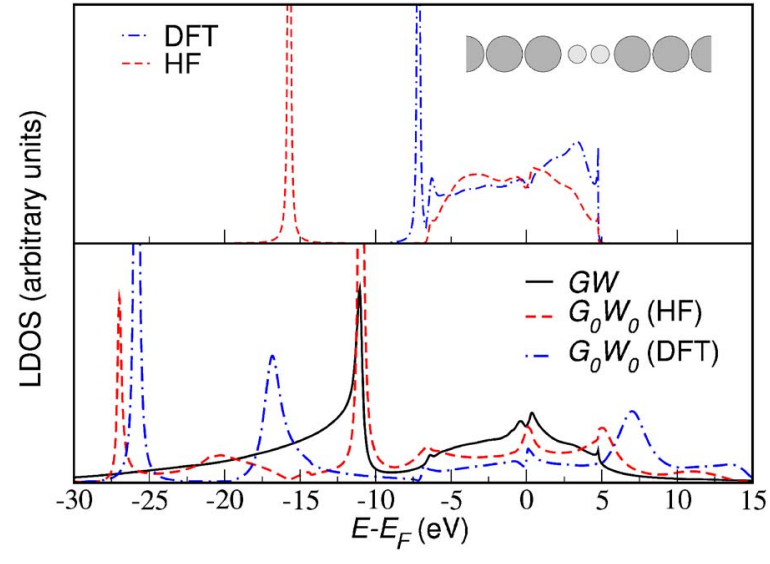

FIG. 3. (Color online) Local density of states at one of the $\mathrm{H}$ orbitals of the $\mathrm{Pt}-\mathrm{H}-\mathrm{H}-\mathrm{Pt}$ contact shown in the inset.

conductance drops dramatically already at biases comparable to $T_{K}$ due to suppression of the Kondo resonance. We note that we do not observe a splitting of the $G W$ Kondo resonance at finite $V .{ }^{24}$ The side peaks in the $d I / d V$ correspond to the central level crossing the chemical potentials. The $G_{0} W_{0}$ conductance is markedly different from the $\mathrm{HF}$ and $G W$ results. At low bias there are unphysical dips in the conductance curve and as $V$ is raised the $d I / d V$ becomes even more unreasonable showing strong negative differential conductance (for this reason we have omitted the $V=4$ curve). This unphysical behavior of the $G_{0} W_{0}$ is a result of its nonconserving nature, and it underlines the necessity of using a conserving approximation such as the self-consistent $G W$ for transport calculations. Within $G_{0} W_{0}$ the violation of current conservation, $\left(I_{L}-I_{R}\right) / I$, increases with $V$ reaching $20 \%$ at $V=0.8$ for certain values of $\varepsilon_{c}$, while it is always negligible in our $G W$ calculations. $^{25}$

As an illustration of the Wannier- $G W$ scheme we consider a molecular hydrogen bridge between infinite atomic $\mathrm{Pt}$ chains, see inset of Fig. 3. Experimentally, the conductance of the hydrogen contact is found to be close to the conductance quantum, $2 e^{2} / h$, and this value has been reproduced by DFT calculations. ${ }^{3}$ Using the plane-wave pseudopotential code DACAPO, we perform DFT calculations for an infinite Pt wire as well as a supercell containing the hydrogen molecule with six Pt atoms on each side. The WFs and KS Hamiltonian of the full system in equilibrium are obtained by combining the two calculations, see Ref. 13 for more details. For the transport calculation we use a central region consisting of the two $s$-like WFs of the hydrogen. Due to the smallness of the matrix elements coupling the two Pt chains across the molecule this suffices to converge the elastic transmission function. Ultimately, the dependence of the $G W$ results on the size of $C$ as well as on the basis set should also be checked. $^{26}$

In the upper panel of Fig. 3 we show the local density of states (LDOS) at one of the two $\mathrm{H}$ orbitals as calculated within DFT using the PW91 xc-functional, as well as selfconsistent HF (in the central region). In DFT the $\mathrm{H}_{2}$ bonding state is a bound state at $-7.0 \mathrm{eV}$ relative to $E_{F}$, while the antibonding state lies at $0.4 \mathrm{eV}$ and is strongly broadened by coupling to the Pt. Moving from DFT to HF the bonding 


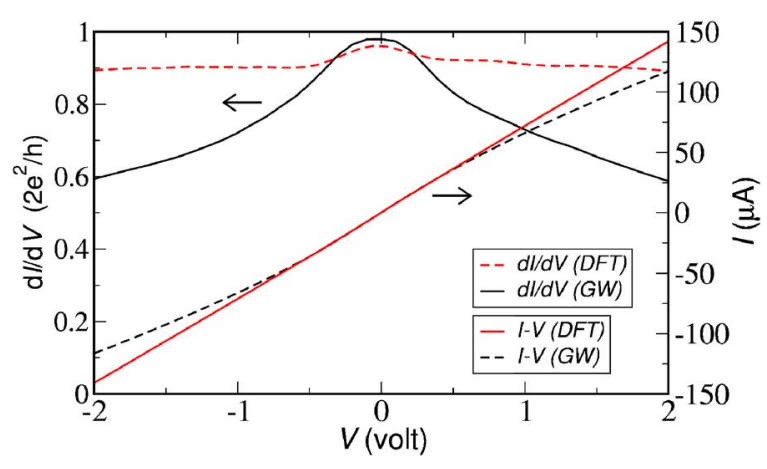

FIG. 4. (Color online) $I-V$ and $d I / d V$ for the hydrogen contact as calculated in DFT(PW91) and self-consistent $G W . V$ is the source-drain bias voltage.

state is shifted down by $\sim 8 \mathrm{eV}$ because for occupied states the exchange potential is more negative than the DFT xcpotential. The same effect tends to drive the half-filled antibonding state down but in this case the resulting increase in the Hartree potential (about $4 \mathrm{eV}$ ) stops it just below $E_{F}$.

In the lower panel of Fig. 3 we show the LDOS calculated in $G W$ as well as $G_{0} W_{0}$ starting from either DFT or HF, i.e., $G_{0}$ is either $G_{\mathrm{DFT}}$ or $G_{\mathrm{HF}}$. The large deviation between the two $G_{0} W_{0}$ results is not surprising given the large difference between $G_{\mathrm{DFT}}$ and $G_{\mathrm{HF}}$. Focusing on the bonding state, the $G_{0} W_{0}$ quasiparticle (QP) energies lie at $-26 \mathrm{eV}$ and $-11 \mathrm{eV}$ for DFT and HF, respectively. Two effects are responsible for this difference. We have $\varepsilon_{\mathrm{QP}}\left(G_{0}\right)=\varepsilon_{\mathrm{HF}}\left(G_{0}\right)$ $+\varepsilon_{\text {corr }}\left(G_{0}\right)$, where $\varepsilon_{\text {corr }}$ is determined by the intersection of the line $\varepsilon-\varepsilon_{\mathrm{HF}}$ with the real part of the correlation selfenergy, $\operatorname{Re} \Sigma_{\text {corr }}^{r}$. Now, $\varepsilon_{\mathrm{HF}}\left(G_{\mathrm{HF}}\right)$ is already $\sim 5 \mathrm{eV}$ larger than $\varepsilon_{\mathrm{HF}}\left(G_{\mathrm{DFT}}\right)$ due, mainly, to the mentioned increase in Hartree energy. Second, it turns out that $\Sigma_{\text {corr }}^{r}\left(G_{\mathrm{HF}}\right)$ is roughly $\Sigma_{\text {corr }}^{r}\left(G_{\mathrm{DFT}}\right)$ shifted down by $\sim 9 \mathrm{eV}$ (note that this corresponds to the difference between the delta peaks in $G_{\mathrm{HF}}$ and $\left.G_{\mathrm{DFT}}\right)$, leading to $\varepsilon_{\text {corr }}\left(G_{\mathrm{DFT}}\right) \approx-6 \mathrm{eV}$ and $\varepsilon_{\text {corr }}\left(G_{\mathrm{HF}}\right) \approx 5 \mathrm{eV} .{ }^{26}$ We are aware that part of this difference could be due to the limited size of the basis.

We notice, that the LDOS results of Fig. 3 can be largely reproduced by including only the second-order $G W$ diagram in the self-energy and thus the higher-order RPA diagrams are less important in this case.

The linear-response conductance has been calculated by applying a small bias of $10 \mathrm{mV}$. All the self-consistent calculations, i.e., DFT, $\mathrm{HF}$, and $G W$, yield a conductance within $10 \%$ of the experimental value of $2 e^{2} / h$. The same holds for $G_{0} W_{0}(\mathrm{HF})$, however, this is somewhat arbitrary as the $G_{0} W_{0}(\mathrm{DFT})$ conductance is only $0.4\left(2 e^{2} / h\right)$. In Fig. 4 we show the fully self-consistent $I-V$ characteristics for DFT and $G W$. The DFT conductance is nearly constant over the bias range (the same holds for the HF calculation, not shown). In contrast the $G W$ conductance falls off at a higher bias due to incoherent scattering described by $\operatorname{Im} \Sigma_{\text {corr }}$ Since $\operatorname{Im} \Sigma_{\text {corr }}\left(E_{F}\right)$ vanishes in equilibrium, the finite-bias conduc- tance suppression is a direct result of the nonequilibrium treatment of correlations.

In conclusion, we have presented a nonequilibrium $G W$ implementation that can be combined with DFT and a localized basis set to model correlated electron transport in nanostructures. Results for the Anderson model and a $\mathrm{Pt}-\mathrm{H}-\mathrm{H}-\mathrm{Pt}$ nanocontact indicate that self-consistency is crucial for $G W$ transport calculations.

The authors thank E. K. U. Gross for stimulating discussions during our stay in his group. The authors were partly supported by the EC 6th Framework No. E NANOQUANTA and SANES project. K.T. acknowledges support from the Danish Natural Science Research Council.

${ }^{1}$ D. Goldhaber-Gordon, Hadas Shtrikman, D. Mahalu, David AbuschMagder, U. Meirav, and M. A. Kastner, Nature (London) 391, 156 (1998).

${ }^{2}$ S. K. Nielsen, Y. Noat, M. Brandbyge, R. H. M. Smit, K. Hansen, L. Y. Chen, A. I. Yanson, F. Besenbacher, and J. M. van Ruitenbeek, Phys. Rev. B 67, 245411 (2003).

${ }^{3}$ D. Djukic, K. S. Thygesen, C. Untiedt, R. H. M. Smit, K. W. Jacobsen, and J. M. van Ruitenbeek, Phys. Rev. B 71, 161402 (2005).

${ }^{4}$ K. Stokbro, J. Taylor, M. Brandbyge, J.-L. Mozos, and P. Ordejón, Comp. Mat. Sci. 27, 151 (2003).

${ }^{5}$ P. Delaney and J. C. Greer, Phys. Rev. Lett. 93, 036805 (2004).

${ }^{6}$ A. Ferretti, A. Calzolari, R. Di Felice, F. Manghi, M. J. Caldas, M. B. Nardelli, and E. Molinari, Phys. Rev. Lett. 94, 116802 (2005).

${ }^{7}$ P. Darancet, A. Ferretti, D. Mayou, and V. Olevano, cond-mat/0611404.

${ }^{8}$ A. Stan, N. E. Dahlen, and R. van Leeuwen, Europhys. Lett. 76, 298 (2006).

${ }^{9}$ T. A. Niehaus, M. Rohlfing, F. Della Sala, A. Di Carlo, and T. Frauenheim, Phys. Rev. A 71, 022508 (2005).

${ }^{10}$ M. S. Hybertsen and S. G. Louie, Phys. Rev. B 34, 5390 (1986).

${ }^{11}$ G. Onida, L. Reining, and A. Rubio, Rev. Mod. Phys. 74, 601 (2002).

${ }^{12}$ K. S. Thygesen, L. B. Hansen, and K. W. Jacobsen, Phys. Rev. Lett. 94, 026405 (2005).

${ }^{13}$ K. S. Thygesen and K. W. Jacobsen, Chem. Phys. 319, 111 (2005).

${ }^{14}$ With our localized basis set we have found that this is sufficient to reproduce Hartree and exchange energies of most molecular orbitals to within $10 \%$.

${ }^{15}$ H. Haug and A.-P. Jauho, Quantum Kinetics in Transport and Optics of Semiconductors, Springer, Berlin (1998).

${ }^{16}$ The last term in Eq. (2) is usually written as $\left(1+G^{r} \sum_{\text {tot }}^{r}\right) G_{0}^{<}\left(1+\sum_{\text {tot }}^{a} G^{a}\right)$, where $G_{0}$ is the Green's function of $H_{C}+V_{H \cdot}{ }^{15}$ Using that $G_{0}^{<}(\omega)$ $=-f_{C}(\omega)\left(G_{0}^{r}-G_{0}^{a}\right)=2 f_{C}(\omega) \eta i$, the equivalence of the two forms is readily shown.

${ }^{17}$ G. Stefanucci and C.-O. Almbladh, Phys. Rev. B 69, 195318 (2004).

${ }^{18}$ Y. Meir and N. S. Wingreen, Phys. Rev. Lett. 68, 2512 (1992).

${ }^{19}$ U. von Barth, N. E. Dahlen, R. van Leeuwen, and G. Stefanucci, Phys. Rev. B 72, 235109 (2005).

${ }^{20}$ C. D. Spataru, L. X. Benedict, and S. G. Louie, Phys. Rev. B 69, 205204 (2004).

${ }^{21}$ In the Kondo regime, the $G_{0} W_{0}$ spectral peak can appear more or less close to $E_{F}$ depending on the values of $\Gamma$ and $U$, whereas the $G W$ peak is always situated very close to $E_{F}$ independent of $U$ and $\Gamma$.

${ }^{22}$ J. A. White, Phys. Rev. B 45, 1100 (1992).

${ }^{23}$ B. Holm and U. von Barth, Phys. Rev. B 57, 2108 (1998).

${ }^{24}$ Y. Meir, N. S. Wingreen, and P. A. Lee, Phys. Rev. Lett. 17, 2601 (1993).

${ }^{25}$ The proof that $I_{L}=I_{R}$ for the present self-consistent $G W$ calculations will be given elsewhere.

${ }^{26}$ See EPAPS No. E-JCPSA6-126-803706 for $G W$-Wannier test results for the HOMO and LUMO levels of an $\mathrm{H}_{2}$ molecule as well as a graphical determination of $\varepsilon_{\text {corr }}$. This document can be reached via a direct link in the online article's HTML reference section or via the EPAPS homepage (http://www.aip.org/pubservs/epaps.html). 